\title{
A Computational Model of Implicit Memory Captures Dyslexics' Perceptual Deficits
}

\author{
Sagi Jaffe-Dax, ${ }^{1}$ Ofri Raviv, ${ }^{1}$ Nori Jacoby, ${ }^{1}$ Yonatan Loewenstein, ${ }^{1,2}$ and Merav Ahissar ${ }^{1,3}$ \\ ${ }^{1}$ Edmond and Lily Safra Center for Brain Sciences, Hebrew University of Jerusalem 9190401, Israel, ${ }^{2}$ Neurobiology Department, Cognitive Sciences \\ Department and the Federmann Center for the Study of Rationality, Hebrew University of Jerusalem, Jerusalem 9190401, Israel, and ${ }^{3}$ Psychology \\ Department, Hebrew University of Jerusalem, Jerusalem 9190501, Israel
}

Dyslexics are diagnosed for their poor reading skills, yet they characteristically also suffer from poor verbal memory and often from poor auditory skills. To date, this combined profile has been accounted for in broad cognitive terms. Here we hypothesize that the perceptual deficits associated with dyslexia can be understood computationally as a deficit in integrating prior information with noisy observations. To test this hypothesis we analyzed the performance of human participants in an auditory discrimination task using a two-parameter computational model. One parameter captures the internal noise in representing the current event, and the other captures the impact of recently acquired prior information. Our findings show that dyslexics' perceptual deficit can be accounted for by inadequate adjustment of these components; namely, low weighting of their implicit memory of past trials relative to their internal noise. Underweighting the stimulus statistics decreased dyslexics' ability to compensate for noisy observations. ERP measurements (P2 component) while participants watched a silent movie indicated that dyslexics' perceptual deficiency may stem from poor automatic integration of stimulus statistics. This study provides the first description of a specific computational deficit associated with dyslexia.

Key words: anchoring deficit; computational neuroscience; event-related potentials; predictive coding; perception; statistical learning

\section{Significance Statement}

This study presents the first attempt to specify the mechanisms underlying dyslexics' perceptual difficulties computationally by applying a specific model, inspired by the Bayesian framework. This model dissociates between the contribution of sensory noise and that of the prior statistics in an auditory perceptual decision task. We show that dyslexics cannot compensate for their perceptual noise by incorporating prior information. By contrast, adequately reading controls' usage of previous information is often close to optimal. We used ERP measurements to assess the neuronal stage of this deficit. We found that unlike their peers, dyslexics' ERP responses are not sensitive to the relations between the current observation and the prior observation, indicating that they cannot establish a reliable prior.

\section{Introduction}

The controversy surrounding the deficits underlying dyslexics' difficulties is still unresolved. The prevailing theory claims that dyslexics' phonological representations, whose adequacy is crucial for efficient usage of the alphabetical code, are impaired (Snowling, 2000). However, dyslexics perform well on some tasks that rely on adequate phonological representations (for review,

\footnotetext{
Received April 2, 2015; revised June 20, 2015; accepted July 17, 2015.

Author contributions: S.J.-D. and M.A. designed research; S.J.-D. performed research; S.J.-D., O.R., and N.J. analyzed data; S.J.-D., O.R., Y.L., and M.A. wrote the paper.

This work was supported by the Israel Science Foundation Grant 616/11, the EPFL-HUJI Brain Collaboration, and the Gatsby Charitable Foundation.

The authors declare no competing financial interests.

Correspondence should be addressed to Merav Ahissar, Psychology Department, Hebrew University of Jerusalem, Jerusalem 9190501, Israel. E-mail: msmerava@gmail.com.

DOI:10.1523/JNEUROSCI.1302-15.2015

Copyright $\odot 2015$ the authors $\quad 0270-6474 / 15 / 3512116-11 \$ 15.00 / 0$
}

see: Ramus and Ahissar, 2012). A computationally motivated imaging study found that dyslexics' task-specific top-down tuning to phonological processing, as revealed in their thalamic response, is impaired (Díaz et al., 2012), suggesting that it may be access to phonological representations which is deficient in dyslexia (Boets et al., 2013; Ramus, 2014). Nevertheless, other studies have found that dyslexics' automatic responses to basic sounds are noisier than those of good readers (Nagarajan et al., 1999; Hornickel and Kraus, 2013). The anchoring deficit hypothesis (Ahissar et al., 2006; Ahissar, 2007) states that dyslexics' deficits stem from poor utilization of stimulus repetitions. In this framework dyslexia does not result from a deficit in stimulus processing (sensation) or alternatively from a deficit in memory representation, but from a deficit in the match between them (similar to the concept of predictive coding; Díaz et al., 2012).

The current study was designed to generalize and quantify the impaired-anchoring hypothesis in a computational model, 
which specifies the experimental conditions that enhance dyslexics' difficulties. A two-tone discrimination task was used because it is simple, quantifiable, and provides a reliable predictor of performance in phonologically related tasks (adults: Amitay et al., 2002; Ben-Yehudah et al., 2004; Oganian and Ahissar, 2012; children: Mengler et al., 2005; Banai and Yifat, 2012; Banai and Ahissar, 2013). Previously, we showed that individuals with dyslexia have difficulties benefitting from simple stimulus repetitions on this task (Ahissar et al., 2006; Ahissar, 2007; Oganian and Ahissar, 2012). Here we used a version of this task that contains no repetitions. Instead, it has richer statistics, which allowed us to analyze the impact of stimulus history on single trials. Specifically, it allowed us to separate the trials in which performance is expected to "benefit" from stimulus history from those trials in which these statistics are expected to hamper performance.

We used an on-line computational model (Raviv et al., 2012), which quantitatively specifies the integration of context on the two-tone discrimination task, to disentangle dyslexics' disabilities. The model posits that rather than comparing the second tone in each trial to the first one, listeners compare the frequency of the second tone to a weighted average of the frequency of the first tone and a memory trace of the frequencies of the tones presented in previous trials. This may allow listeners to reduce the disruptive effect of noise in the internal representation of the first tone (e.g., noise added in the retention interval) by combining this noisy representation with that of previous trials in a way that qualitatively resembles Bayesian reasoning ( $\mathrm{Lu}$ et al., 1992; Preuschhof et al., 2010; Ashourian and Loewenstein, 2011). Note, however, that a Bayesian inference specifies exactly how the prior distribution should be integrated in perception. By contrast, the prior distribution is not explicitly learned in our model, and the extent to which previous trials affect perception is a parameter. Using the model we conclude that dyslexics' poorer perceptual performance is associated with suboptimal incorporation of prior knowledge in perception.

To further characterize the processes underlying the integration of the stimulus statistics in perception, we measured ERP responses that are sensitive to stimulus statistics (the $\mathrm{P} 2$ component; Tremblay et al., 2001, 2010). Unlike controls' P2, dyslexics' P2 did not show such sensitivity.

These results pave the way for a better understanding of dyslexics' perceptual deficits as a computational impairment related to the learning and incorporation of prior sensory information.

\section{Materials and Methods}

Participants. Twenty-eight native Hebrew speakers (14 dyslexics and 14 good readers), who are students at the Hebrew University (mean age = 25.4 years; STD $=2.2 ; 18$ females), were considered in this study. Recruitment was based on ads at the Hebrew University. Monetary compensation for participation was according to standard student rates. The study was approved by the Hebrew University Committee for the Use of Human Subjects in Research. All dyslexic participants (except one, whose reading, phonological, and reasoning profile matched the profile of the other dyslexic participants) had been diagnosed before the study by clinicians using a standard didactic test approved by the Israeli Ministry of Education. Six participants, with $>2$ years of formal musical education were excluded, so that musical training would not be a major contributor to their pitch sensitivity (Micheyl et al., 2006; Parbery-Clark et al., 2011). Two participants with poor Block Design scores (lower than a normalized score of 7) were also excluded from the study. All participants filled out a questionnaire regarding any neurological or psychiatric disorders. None of the participants reported any such disorders.

Procedure. All participants were administered three sessions on three different days. In session 1 participants were administered a series of cognitive assessments. In session 2, ERPs were recorded. Participants watched a silent movie while a series of stimuli was presented to them. In session 3 the same series of stimuli was presented, and participants actively engaged in the frequency discrimination task.

General assessments. General cognitive abilities were assessed using two standard tasks. (1) Nonverbal reasoning ability was measured with the Block Design, a standard test for assessing visuospatial reasoning (WAISIII; Wechsler, 1997). (2) Short-term verbal memory was evaluated with the standard Digit Span task (forward and backward; Hebrew version of WAIS-III; Wechsler, 1997).

Phonological skills. Phonological skills were assessed using two types of phonological tasks. (1) Phonological decoding was tested when single pseudoword and real-word reading were assessed using two standard Hebrew lists designed by Deutsch and Bentin (1996). One list contains 24 punctuated Hebrew words and the other contains 24 punctuated pseudowords; i.e., words with Hebrew morphology but no meaning. Both accuracy and rate were scored. (2) Phonological awareness was assessed using the Spoonerism task (MacKay, 1970; Möller et al., 2007). Participants heard (Hebrew) word pairs and were asked to switch the first phonemes of the two words and respond vocally (e.g., /laila tov/, "good night" in Hebrew, should be switched to /taila lov/). Both accuracy and rate were scored. We merged accuracy and speed in all phonological tests into a combined phonological score by averaging the $z$-score (relative to the control group distribution) within each subject.

Stimulation procedure for two-tone frequency discrimination. Participants were presented with sequences (blocks) of tone pairs (50 ms, $70 \mathrm{~dB}$ each tone; $620 \mathrm{~ms}$ intertone intervals and $1380 \mathrm{~ms}$ interpair intervals). They were asked to indicate which of the two tones in each pair (i.e., trial) had a higher pitch. No feedback was provided.

Participants were presented with a fixed easy-to-difficult sequence of stimuli based on the average sequence of frequency differences that were given to participants (students, adequate readers with no, or only minor, musical background) in an adaptive staircase procedure (three down, one up), which converges at $\sim 80 \%$ correct (Nahum et al., 2010). Consequently, the average performance was similar in the two protocols. This pseudoadaptive sequence of tone pairs was chosen in order to use the same easy-to-difficult sequence of stimuli for all subjects, under both the passive and active conditions.

Each participant performed 700 trials in two separate blocks. (1) In the No-Reference protocol, on each trial, a tone was chosen randomly from 800 to $1200 \mathrm{~Hz}$. The other tone was determined according to the frequency difference that was preassigned to that trial and could be higher or lower than the randomly chosen tone. The order of the tones was random. This block consisted of 300 trials. The difference between the two tones began at $25 \%$ and decreased gradually to $18 \%$. (2) In the Reference-Interleaved condition, one of the tones was $1000 \mathrm{~Hz}$ in all trials (i.e., Reference tone) and the other tone was determined according to the frequency difference that was preassigned to that trial. The order of the tones was predetermined: odd number trials, Reference first; even number trials, Reference second. This block consisted of 400 trials. The difference between the two tones began at $25 \%$ and decreased gradually to $5-7 \%$. The order of the blocks was counterbalanced across participants.

Parameter estimation of the implicit memory model. We modeled the responses made by the participants using the Implicit Memory Model (IMM; Raviv et al., 2012). According to this model, choices in each trial are determined by the difference between the frequency of the second tone and a weighted average $\left(M_{1}\right)$ of the noisy memory of present and past frequencies of the first tone. Formally,

$$
a(t)=\operatorname{sgn}\left(M_{1}(t)-f_{2}(t)\right),
$$

where $a(t)$ denotes the choice of the participant on trial $t$, sgn is the sign function, $f_{2}(t)$ is the frequency of the second tone on trial $t$, and

$$
M_{1}(t)=\eta M_{1}(t-1)+(1-\eta)\left(f_{1}(t)+z(t)\right),
$$

where $f_{1}(t)$ is the frequency of the first tone on the trial, $z(t)$ is an independent Gaussian random variable with zero mean and variance $\sigma^{2}$, and we assume resetting of the initial conditions $M_{1}(t=1)=f_{1}(t=1)+z(t=1)$ 
(Shteingart et al., 2013). This model is characterized by two parameters, $\sigma$ and $\eta$. The first parameter $\sigma$ denotes the level of internal noise in the process of "sensing and memorizing" the first tone on each trial. The second parameter $\eta$ denotes the weight of previously stored stimuli in the current comparison.

Therefore, the probability to respond "first tone higher" is as follows:

$$
\operatorname{Pr}[“ 1 ”]=\Phi\left(\frac{M_{1}(t)-f_{2}(t)}{\sigma_{e}(t)}\right)
$$

where $\Phi$ is the cumulative normal distribution function and $\sigma_{e}(t)$ is the effective response variance at trial $t$, which depends on $\sigma, \eta$, and $t$ :

$$
\sigma_{e}=\sigma \sqrt{\eta^{2 t-2}+\left(1-\eta^{2 t-2}\right) \frac{1-\eta}{1+\eta}}
$$

For each participant, we estimated the two parameters, $\eta$ and $\sigma$, which minimize the squared distance between the predicted response probabilities of the model and the observed responses in the frequency discrimination task. To assess the reliability of this estimate, we bootstrapped the trials for each participant by 1000 samples of 300 trials with replacement and re-estimating parameters.

Calculating the optimal $\eta$. The optimal $\eta$ is defined as the value of $\eta$ that minimizes the probability of an error in the stimulation schedule for a fixed value of $\sigma$.

We defined mental difference $(D(t))$ and correct difference $(C(t))$ on each trial as follows:

$$
\begin{aligned}
& D(t)=f_{2}(t)-M_{1}(t) \\
& C(t)=f_{2}(t)-f_{1}(t) .
\end{aligned}
$$

Thus the probability to make a correct decision on each trial is as follows:

$$
\operatorname{Pr}[\text { correct }]=\operatorname{Pr}[\operatorname{sign}(D(t))=\operatorname{sign}(C(t))]
$$

According to Sheppard's Median Dichotomy Theorem (Sheppard, 1899; Kendall et al., 1987, their p. 482):

$$
\operatorname{Pr}[\operatorname{sign}(D(t))=\operatorname{sign}(C(t))]=\frac{1}{2}+\frac{1}{\pi} \sin ^{-1}\left(\rho_{m}\right),
$$

where $\rho_{m}=\frac{\operatorname{cov}(D, C)}{\sqrt{\operatorname{var}(D) \operatorname{var}(C)}}$ is the correlation between the mental difference and the correct difference.

The probability of a correct response is monotonous over $\rho_{m}$; thus it is sufficient to maximize $\rho_{m}$ over $\eta$ :

$$
=\frac{\left(2-\eta+(\eta-2) \rho_{f}\right) \sigma_{f}^{2}}{\sqrt{\left(\begin{array}{c}
\sigma_{f}^{2}+2 \sigma_{f}^{2}(\eta-1) \rho_{f}+\eta^{2}\left(\frac{1-\eta}{1+\eta}\left(\sigma_{f}^{2}+\sigma^{2}\right)\right) \\
+(1-\eta)^{2}\left(\sigma_{f}^{2}+\sigma^{2}\right)
\end{array}\right)\left(2\left(1-\rho_{f}\right) \sigma_{f}^{2}\right)}}
$$

where $\rho_{f}=\frac{\operatorname{cov}\left(f_{1}, f_{2}\right)}{\sqrt{\operatorname{var}\left(f_{1}\right) \operatorname{var}\left(f_{2}\right)}}$ is the correlation between the two tones and $\sigma_{f}^{2}$ is the variance of $f_{1}$ (the overall variance of the frequencies of the first tones), which is also equivalent to the variance of $f_{2}$ (the same marginal distributions for the frequencies of the first and the second tones).
The optimal $\eta$ is a solution to the equation:

$$
\frac{\partial \rho_{m}}{\partial \eta}=0
$$

Resulting in

$$
\eta^{*}=\frac{1}{6}\left(\frac{2 \sigma^{2}}{\sigma_{f}^{2} \rho_{f}}+\frac{2^{4 / 3}\left(\sigma_{f}^{4}\left(\rho_{f}-9\right) \rho_{f}-7 \sigma_{f}^{2} \rho_{f} \sigma^{2}+\sigma^{4}\right)}{\sigma_{f}^{2} \rho_{f} A}+\frac{2^{2 / 3}}{\sigma_{f}^{2} \rho_{f}} A-4\right)
$$

where

$$
\begin{gathered}
A=\sqrt[3]{\begin{array}{c}
2 \sigma_{f}^{6} \rho_{f}^{2}\left(27+\rho_{f}\right)+3 \sigma_{f}^{4} \rho_{f}\left(20 \rho_{f}-9\right) \sigma^{2} \\
-21 \sigma_{f}^{2} \rho_{f} \sigma^{4}+2 \sigma^{6}+9 \sigma_{f}^{2} \rho_{f} B
\end{array}} \\
B=\sqrt{\begin{array}{c}
\left(\sigma_{f}^{2}+\sigma^{2}\right)\left(4 \sigma_{f}^{6} \rho_{f}\left(3+\rho_{f}\right)^{2}+12 \sigma_{f}^{4} \rho_{f}\left(1+3 \rho_{f}\right) \sigma^{2}\right. \\
-3 \sigma_{f}^{2}\left(1+4 \rho_{f}\right) \sigma^{4}+\sigma^{6}
\end{array}} .
\end{gathered}
$$

Since this computation assumes an infinite number of trials, the solution is only an asymptotic approximation of the optimal $\eta$. To verify its proximity to the optimal $\eta$, with a finite number of trials, we ran numerical simulations. We estimated the $\eta$ that yielded the highest accuracy rate in the actual sequence of trials used in the experiment for various values of $\sigma$ and compared the two solutions. The numerical and analytical calculations nearly overlapped.

We then estimated the optimal impact of implicit memory given participants' estimated $\sigma$; i.e., for each participant we found $\eta^{*}$ - the $\eta$ that would maximize success on the auditory task. We defined inadequacy, or suboptimality, of the implicit memory weighting of each participant as the difference between $\eta^{*}$ and the estimated $\eta$.

$E R P$ recordings and analyses. Electrophysiological activity was recorded in a sound-attenuated room while participants heard the tone sequences either passively (session 2 ) or while actively performing the task (session 3). Sounds were produced using MATLAB (The MathWorks) and were presented by E-Prime 1.1 software (Psychology Software Tools). The EEG was recorded from 32 active $\mathrm{Ag}$ - $\mathrm{AgCl}$ electrodes mounted on an elastic cap using the BioSemi ActiveTwo tools and recording software. Electrode sites were based on the 10-20 system (American Electroencephalographic Society, 1991). Two additional electrodes were placed over the left and right mastoids. Horizontal EOG was recorded from two electrodes placed at the outer canthi of both eyes. Vertical EOG was recorded from electrodes on the infraorbital and supraorbital regions of the right eye in line with the pupil.

EEG and EOG signals were sampled at $256 \mathrm{~Hz}$, amplified and filtered with an analog bandpass filter of $0.16-100 \mathrm{~Hz}$. Off-line analysis was performed using Brain Vision Analyzer 1.05 software (Brain Products). The EEG signal was digitally bandpass filtered between 1 and $30 \mathrm{~Hz}$ to remove large drifts in signal and high-frequency noise. Independent component analysis was trained on the entire length of each block to identify components that reflect eye blink or eye movement-evoked electrical activity. An eye-related component was identified by its time correlation with the occurrence of blinks or saccades. This relationship between the identified component and eye blink activity was verified by controlling the component's scalp distribution that was typical of eyerelated electrical activity. Data were referenced to the nose channel to remove external electrical influence. Artifact rejection was applied to the nonsegmented data according to the following criteria: any data point with EOG or EEG $> \pm 100 \mu \mathrm{V}$ was rejected along with the data $\pm 300 \mathrm{~ms}$ around it. In addition, if the difference between the maximum and the minimum amplitudes of two data points within an interval of $50 \mathrm{~ms}$ exceeded $100 \mu \mathrm{V}$, data $\pm 200 \mathrm{~ms}$ around it were rejected. Finally, if the difference between two adjacent data points was $>50 \mu \mathrm{V}$, the data \pm 300 $\mathrm{ms}$ around it were rejected. Trials containing rejected data points were omitted from further analysis (average omission of 17 trials per subject).

For ERP averaging across trials, the EEG was parsed to $2000 \mathrm{~ms}$ epochs starting $500 \mathrm{~ms}$ before the onset of the first stimulus in each pair and 
Table 1. Participants' general cognitive and phonological skills

\begin{tabular}{|c|c|c|c|}
\hline Test & $\begin{array}{l}\text { Control } \\
\text { (STD) } \\
N=14\end{array}$ & $\begin{array}{l}\text { Dyslexic } \\
\text { (STD) } \\
N=14\end{array}$ & $\begin{array}{l}\text { Mann- } \\
\text { Whitney } \\
z \text {-value }\end{array}$ \\
\hline \multicolumn{4}{|l|}{ General cognitive } \\
\hline Block Design Norm & $12.4(2.3)$ & $10.8(2.3)$ & 1.7 \\
\hline Digit Span Norm & $11.6(3.4)$ & $7.4(2.2)$ & $3.2^{* *}$ \\
\hline \multicolumn{4}{|l|}{ Phonological decoding speed (items/min) } \\
\hline Single-word reading rate & $104.5(33.1)$ & $62.7(18.4)$ & $3.5^{* * *}$ \\
\hline Pseudoword reading rate & $59.7(16.4)$ & $29.4(9)$ & $4.3^{* * *}$ \\
\hline \multicolumn{4}{|c|}{ Phonological decoding accuracy (fraction correct) } \\
\hline Single-word reading accuracy & $1(0)$ & $0.9(0.1)$ & $3.7^{* * *}$ \\
\hline Pseudo-word reading accuracy & $0.9(0.1)$ & $0.6(0.2)$ & $4^{* * *}$ \\
\hline \multicolumn{4}{|l|}{ Phonological awareness } \\
\hline Spoonerism rate (items/min) & $10.9(4)$ & $5.1(2)$ & $3.7^{* * *}$ \\
\hline Spoonerism accuracy (fraction correct) & $0.9(0.1)$ & $0.7(0.3)$ & $2.6^{* *}$ \\
\hline
\end{tabular}

${ }^{* *} p<0.01,{ }^{* * *} p<0.001$.

averaged separately for each electrode. The baseline was adjusted by subtracting the mean amplitude of the prestimulus period (500-150 ms before the onset of the first stimulus in the trial) of each ERP from each data point in the epoch. The prestimulus baseline period was calculated from this time interval to exclude effects of anticipatory responses that precede informative anticipated stimuli, such as contingent negative variation (CNV; Walter et al., 1964).

ERP analysis was based on the epochs that were recorded with electrode $\mathrm{Cz}$ (at the vertex of the scalp) after they were processed as described above. This electrode measured the most prominent response to the auditory stimuli. Each participant's data (accumulated during the two blocks) were analyzed separately.

\section{Results}

\section{Participants' cognitive profile}

Dyslexics' performance did not differ from controls' performance on general reasoning skills as measured by the Block Design (Snowling, 2000). However, as expected, their performance was poorer than the controls' performance on the measures of verbal memory and phonological skills (see Table 1). All of the dyslexic participants were poorer decoders than all of the control participants. Specifically, the combined phonological score (average $z$-scores across all phonological tasks) of the best reader in the dyslexic group was 0.3 SDs lower than that of the poorest reader in the control group.

\section{The effect of the stimulus statistics on performance in frequency discrimination}

To evaluate the impact of the stimulus statistics on perception parametrically, we used the two-tone frequency discrimination task. On each trial, participants are presented with two sequentially presented pure tones and are instructed to indicate which had a higher pitch (Fig. $1 A-C$; see also Materials and Methods). The frequencies of the two stimuli were drawn from a broad distribution, a protocol we denoted as the No-Reference protocol. Although frequency discrimination tasks are traditionally used as an assessment of low-level sensory bottlenecks, we have shown that in this (No-Reference) protocol, which is devoid of a repeating standard tone, performance is substantially affected by the statistics of previous trials (Raviv et al., 2012). When the two tones are higher than the average, performance has been shown to be better when the second tone is higher than the first. Similarly, when the two tones are lower than the average, findings have indicated that performance is better when the second tone is also lower than the first (Romo et al., 2002; Hairston and Nagarajan, 2007; Alcalá-Quintana and GarcíaPérez, 2011; Raviv et al., 2012). This result can be understood in a
Bayesian framework where participants use prior information about the distribution of frequencies to compensate for noise in the representation of the memorized tone. Loosely speaking, this computation results in a "contraction" of the representation of the first tone to the mean frequency in the experiment.

Based on these findings, we divided the trials according to the sign of the predicted contribution of the statistics of the experiment to the trial. Specifically, we divided the trials into three types, based on their relationship to the mean frequency. Bias+ trials were defined as those trials in which the experiment's statistics was predicted to improve performance. Namely, (1) the frequencies of the two tones were either both higher than the mean frequency or both lower than the mean frequency and (2) the frequency of the second tone was more extreme than that of the first tone (higher when the two tones were above average and lower when they were below average; Fig. $1 A, D, E$, yellow zones). In these trials the contraction of the first tone toward the mean increases its perceived difference from the second tone, and was expected to improve performance. In contrast, Bias - trials were trials in which the statistics was predicted to hamper performance. Namely, when the two tones were above the average, the second tone was lower than the first and when they were lower than the average, the second tone was higher than the first (Fig. $1 B, D, E$, gray zones). In these trials, contracting the first tone toward the mean frequency decreased its perceived difference from the second tone and was expected to be detrimental to performance. Bias0 trials (Fig. $1 C, D, E$, white zones) were trials in which the first and the second tone flanked the mean frequency.

In line with a previous study (Raviv et al., 2012), we found that trial type had a marked effect on the ability of controls to discriminate the two tones. The probability that controls would correctly discriminate the frequencies of the two stimuli in the Bias+, Bias0, and Bias - regions spanned a broad range of 92, 81.8, and $54.9 \%$, respectively. This was found despite the fact that the experiment was designed in a way that there was almost no difference in the "objective" difficulty of the three regions (quantified as the absolute difference between the frequencies of the two tones on a logarithmic scale depicted as the distance of the points from the diagonal in Fig. $1 D, E$ ). When quantifying performance in terms of $d^{\prime}$, we found that the median $d^{\prime}$ was significantly better in the Bias+ regions than in the Bias0 regions [Median (interquartile range - IQR), Bias $+d^{\prime}: 3.2$ (2); Bias0 $d^{\prime}: 2$ (1.9); Wilcoxon test, $p<0.005$ ] and was significantly better in the Bias0 regions compared with the Bias - regions (Bias $-d^{\prime}: 0$ (2.4); Wilcoxon test, $p<0.001)$. In fact, the control group's performance in the Bias - regions was not significantly different from chance (Wilcoxon test, $p=0.55$ ).

In contrast, dyslexics' performance in the Bias - region was significantly above chance (58.2\% Wilcoxon test, $p<0.05)$, whereas their accuracy in the Bias + regions was lower than that of the controls $(72.4 \%)$. Their performance in the Bias0 region was intermediate $(70.7 \%)$. When quantifying their performance in terms of $d^{\prime}$, their median $d^{\prime}$ was not significantly better in the Bias + regions than in the Bias0 regions [Median (IQR), Bias $+d^{\prime}$ : 1 (1); Bias0 $d^{\prime}: 1$ (1); Wilcoxon test, $p=0.36$ ], although it was significantly better in the Bias 0 regions than in the Bias - regions [Bias - $d^{\prime}: 0.5$ (0.8); Wilcoxon test, $p<0.02$ ].

To test whether dyslexics' sensitivity to trial type was significantly less than that of the controls, we assessed the performance of each participant individually. This is illustrated in Figure 2, where the value of $d^{\prime}$ of each participant in each region (Bias + , Bias0, and Bias-) is plotted for all participants (Fig. 2A, Controls; $B$, Dyslexics). To quantify the sensitivity to the prior distribution 

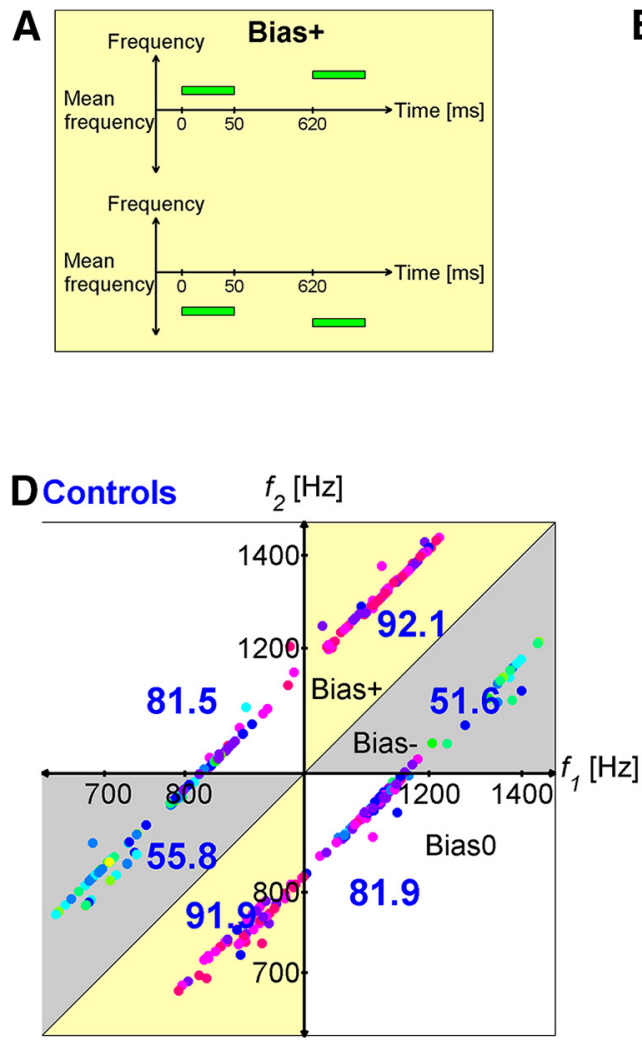
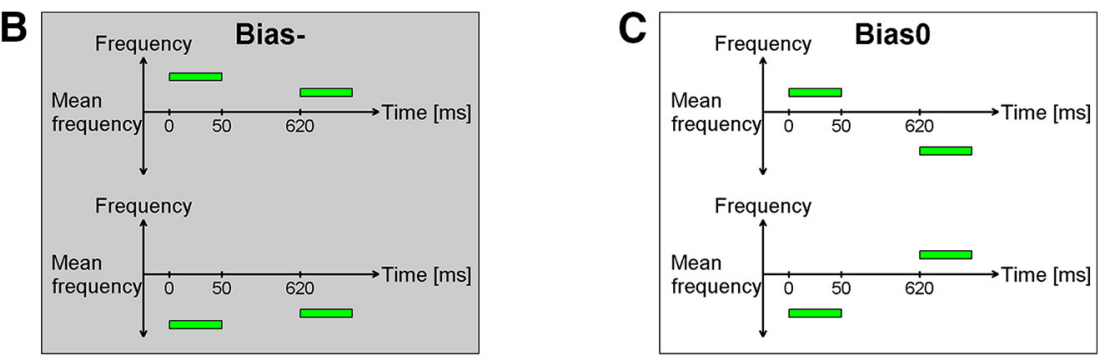

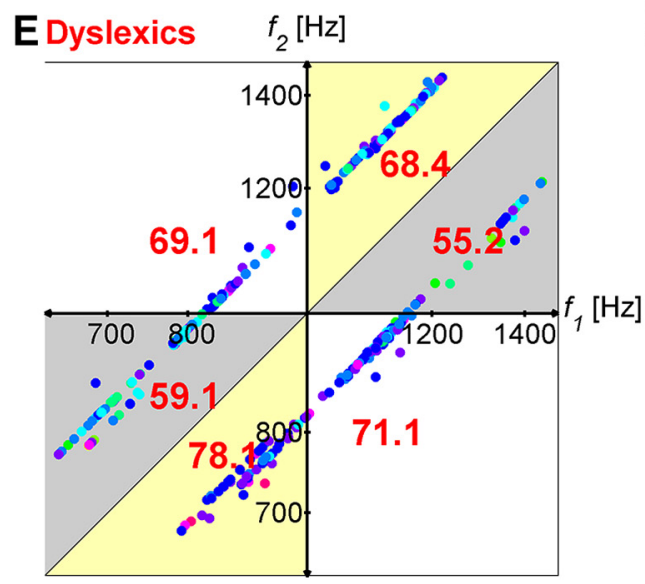

\section{Cross subject single trial accuracy (\% correct)}

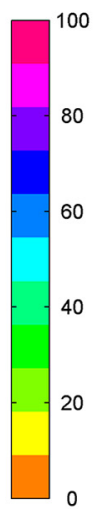

Figure 1. Performance of Controls and Dyslexics differentially depend on trial type. $\boldsymbol{A}-\boldsymbol{C}$, Schematic examples of the three types of trials: in Bias + trials the first tone is closer to the mean $(\boldsymbol{A})$, in Bias - trials the first tone is farther from the mean $(\boldsymbol{B})$, and in Bias0 trials the two tones flank the mean $(\boldsymbol{C})$. $\boldsymbol{D}-\boldsymbol{E}$, Mean performance (percentage correct) of Controls $(\boldsymbol{D})$ and Dyslexics $(\boldsymbol{E})$ in the six subregions of trial types, plotted on the frequency plane of the second tone $f_{2}$ as a function of the first tone $f_{1}$. Bias + zones (denoted in yellow) are above the diagonal when both tones are above the mean frequency (second tone is higher) and below the diagonal when both tones are below the mean frequency (second tone is lower). Bias - regions (denoted in gray) are complementary with respect to the diagonal, and Bias 0 trials (denoted in white) are those trials associated with the two remaining quarters. Each dot denotes $f_{1}$ and $f_{2}$ of a trial (tested across individuals). The color of each dot denotes the cross-subject average performance for that pair of stimuli. Numbers denote the average percentage correct in each region.

A

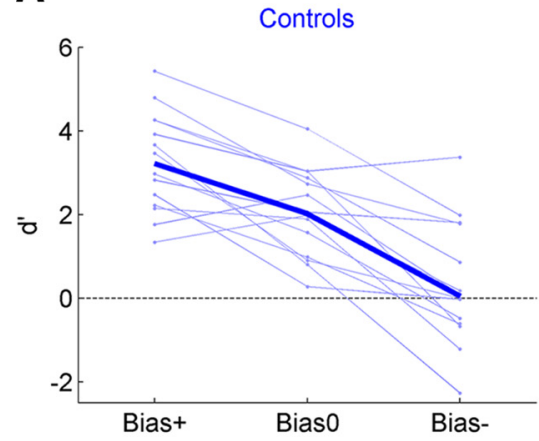

B

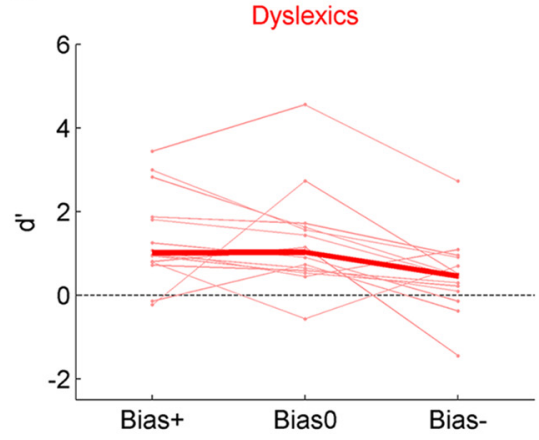

C

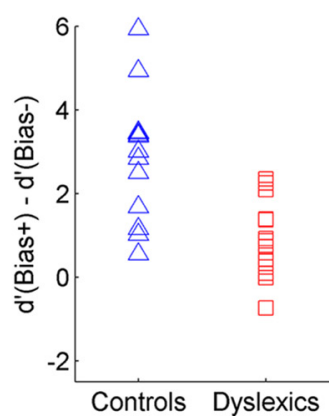

Figure 2. Individual $d^{\prime}$ 's in the three regions, showing their sensitivity to the stimulus statistics. $\boldsymbol{A}, \boldsymbol{B}$, Performance $\left(d^{\prime}\right)$ of Controls $(\boldsymbol{A} ; N=14)$ and Dyslexics $(\boldsymbol{B} ; N=14)$ in Bias,+ Bias 0, and Bias - trials. Thick lines denote medians. Thin lines denote individual performance. C, Difference between Bias $+d^{\prime}$ and Bias $-d^{\prime}$ for each participant (Controls in blue and Dyslexics in red). In both groups, performance in the Bias + regions was generally better than in the Bias - region ( $d^{\prime}$ of Bias $+>d^{\prime}$ of Bias0 $>d^{\prime}$ of Bias - ; Friedman tests, Controls: $p<0.00005$; Dyslexics: $p<0.005$ ). But Controls were more sensitive to the prior distribution than Dyslexics (Group $\times$ Condition interaction; Mann-Whitney test for interaction of Bias + vs Bias - and Controls vs Dyslexics, $z=3.5$, $p<0.001)$.

we computed the difference between the values of $d^{\prime}$ in the Bias+ and Bias - regions for each participant (Fig. 2C). Overall, dyslexics' sensitivity, as measured by this difference, was significantly smaller than controls' sensitivity [Mann-Whitney test for Condition (Bias + vs Bias -$) \times$ Group (controls vs dyslexics) interaction: $z=3.5, p<0.001]$. These results further indicate that dyslexics were less influenced by the statistics of the experiment than controls. Note that the dyslexics' but not the controls' performance was significantly above chance level even in the Bias- region, indicating that their reduced sensitivity to the prior distribution did not stem from a "floor effect." That is, dyslexics' overall poorer performance cannot account for the smaller difference between their performance on the Bias + and Bias - trials, since they performed better than chance in the Bias- region.

\section{Modeling the effect of statistics with the IMM}

The division of the trials into Bias + , Bias-, and Bias 0 drew on the Bayesian framework, which specifies how performance in a 


\section{A simulated controls}

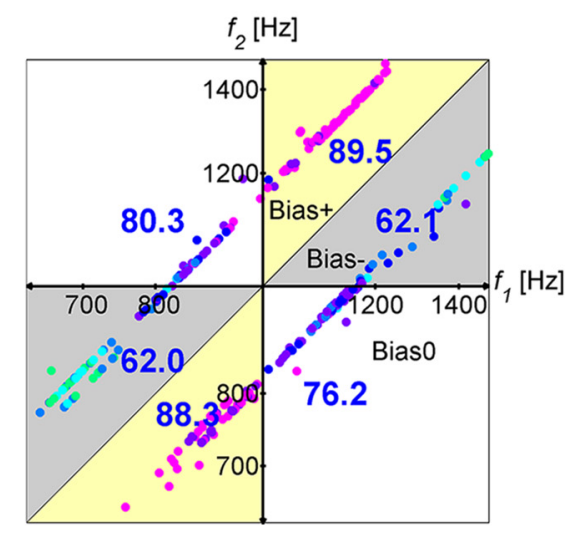

BSimulated dyslexics

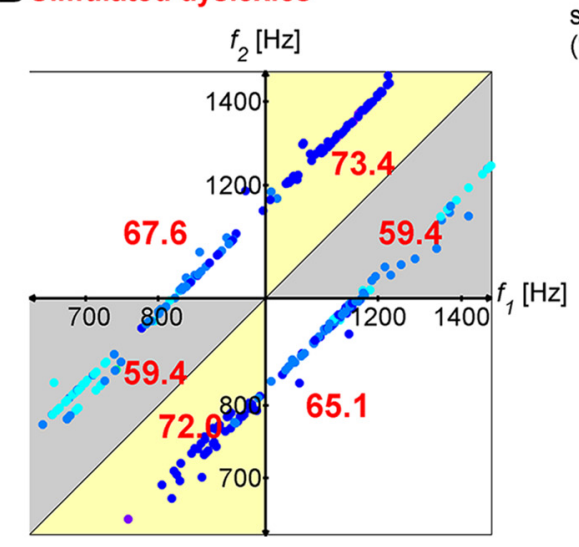

Cross simulated model single trial accuracy (\% correct)

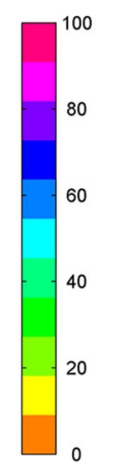

Figure 3. Simulating the experiment with the IMM model produces similar results to those measured experimentally (shown in Fig. 1). $\boldsymbol{A}, \boldsymbol{B}$, Mean performance (percentage correct) of Simulated controls $(\boldsymbol{A})$ and Simulated dyslexics $(\boldsymbol{B})$ in the six subregions of trial types plotted on the $\left[f_{1}, f_{2}\right]$ plane. Bias + zones are denoted in yellow, Bias - in gray, and Bias 0 in white. Each dot denotes $f_{1}$ and $f_{2}$ of a trial (tested across simulated individuals). The color of each dot denotes the average cross-simulation performance for that pair of stimuli. Numbers correspond to the percentage correct in each region.

noisy system can benefit maximally from accumulating environmental statistics and incorporating it into its decision making. As shown above, human performers use such statistics even in simple two-tone discriminations. Nevertheless, this incorporation of the statistics deviates from the predictions of an optimal Bayesian performer (Raviv et al., 2012). To quantify listeners' performance, we used a simplified model (IMM) in which listeners do not know the full distribution of the stimulus statistics. Rather, they weigh past trials using an exponentially decaying function (Raviv et al., 2012).

The model (Eq. 2) is characterized by two parameters: $\eta$, the contribution of previous trials ( $\eta=0$, no contribution; the larger the value of $\eta$, the larger the contribution), and $\sigma$, the level of internal noise (on a scale of percentage difference between the two tones: the larger the value of $\sigma$ the noisier the within-trial representation). We used the IMM to estimate the values of $\eta$ and $\sigma$ for each participant. We tested the model by simulating it on the same task using the estimated parameters. Qualitatively, the differential performance in the three trial types and the different performance level of dyslexics and controls was captured by the model (Fig. 3). Quantitatively, the difference in performance between the Bias + and Bias- regions was slightly larger in the experiment than in the model, in particular for the control participants.

One prediction of the Bayesian framework is that the effect of the stimulus statistics on behavior should increase when the level of internal noise increases. Qualitatively, this prediction is intuitive. If the representation of the stimuli is noiseless, performance cannot be improved by incorporating prior information. However, if the representation of a stimulus is noisy, prior information would be useful, and the noisier the representation, the larger the weight that should be given to this earlier in the discrimination task. One study in fact showed that in the visual modality, increasing the level of internal noise enhanced the contribution of prior knowledge to perception and decision making (Ashourian and Loewenstein, 2011).

The IMM model makes no assumptions regarding the relationships between participants' $\eta$ and $\sigma$. Nevertheless the model can be used to determine the extent to which participants' weighting of previous trials (their $\eta$ ) was close to optimal given their within-trial noise $(\sigma)$ and the stimulus statistics (in the sense of maximizing their success rate; Fig. $4 A$, green).
Analysis of the parameters characterizing our participants indicated that on average, the estimated value of $\sigma$ was higher among the dyslexic participants [Median (IQR), controls: $\sigma=35$ (40)\%; dyslexics: $\sigma=98$ (213)\%; Mann-Whitney test, $z=2.2$, $p<0.05]$. Given their higher $\sigma$, the optimal model solution predicted that the dyslexics' $\eta$ should also be higher than the control's $\eta$. However, the dyslexics' $\eta$ was similar to that of the controls [Median (IQR), controls: $\eta=0.52$ (0.5); dyslexics: $\eta=$ 0.41 (0.53); Mann-Whitney test, n.s.].

As shown in Figure $4 A$, controls' $\eta$ s (blue squares) were nearly optimal (green line) given their $\sigma \mathrm{s}$, indicating that their weighting of the history was similar to that of an "ideal listener" (in the framework of the IMM). In contrast, dyslexics' $\eta$ s were lower than expected given their $\sigma$ s (red squares). To quantify this group difference, we calculated the difference between the optimal and the actual $\eta$ for each participant. We found that Dyslexics' underweighting of implicit memory was significantly larger than the Controls' underweighting (Fig. 4B; Mann-Whitney test, $z=2.6$, $p<0.01)$. Hence, given their internal noise level, Dyslexics, in contrast to Controls, do not give sufficient weight to prior information.

Note that our simple, two-parameter IMM model only assumes within-trial noise. In this framework, the dyslexics' underweighting of the stimulus statistics was suboptimal. An alternative account would posit that dyslexics' memory is noisy. However, introducing this to the model would require the addition of a third parameter, whereas our model accounts well for controls' performance. On the other hand, we could assume optimal weighting of the history (i.e., the weighting of the history is determined by the stimulus statistics) and replace $(\eta)$ with a parameter that estimates the noise of previous trials (memory noise). However, this would require an additional optimality assumption.

Together, the findings show that in the framework of IMM, controls' weighting of past events was nearly optimal. In contrast, dyslexics' weighting of these past events was too low, indicating a deficit in adequate incorporation of prior knowledge into perception.

\section{ERP results}

The IMM model posits that in the two-tone discrimination task, the listener compares the frequency of the second tone to a linear combination of the frequencies of the first tone in the current and 

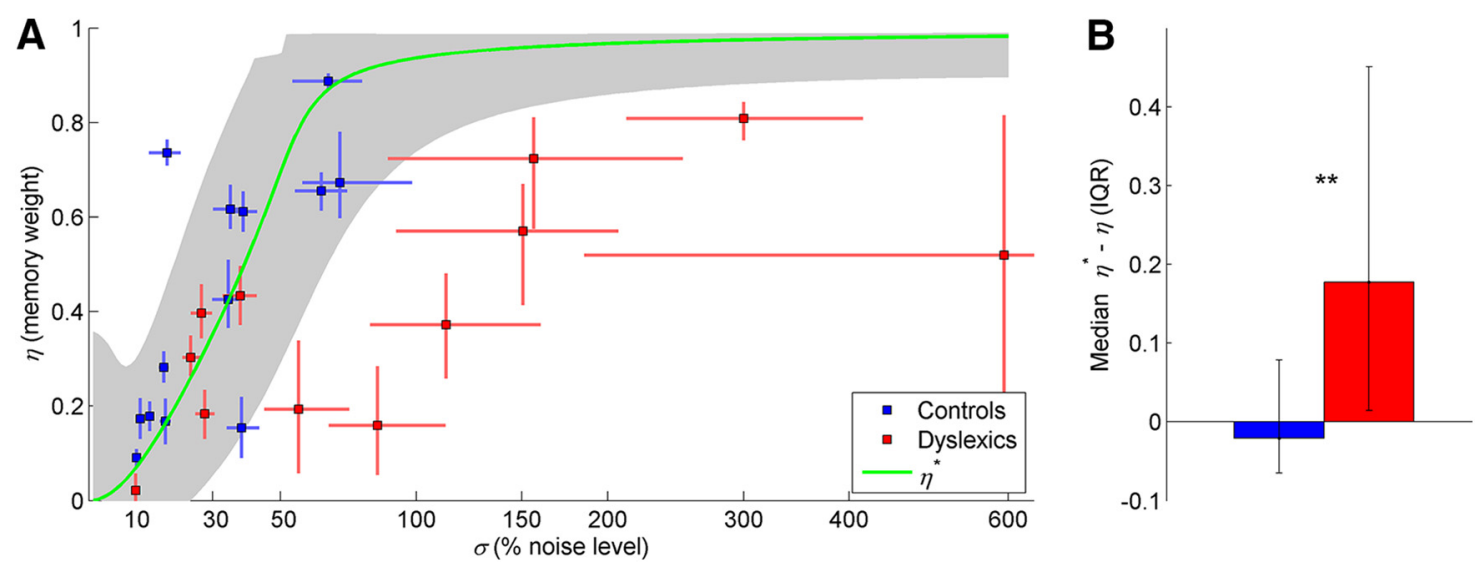

Figure 4. Estimated parameters of the IMM for controls and dyslexics. $A$, Estimated values of $\eta$ (which determine the proportion of implicit memory in the current representation of $f_{1}$ ) as a function of the estimated values of $\sigma$ (percentage of internal noise with respect to the difference between the tones within the trial) of controls (blue; error bars denote bootstrapped IQR) and dyslexics (red). For all values of $\sigma$ the optimal value of $\eta$ (the value of $\eta$ that minimizes the probability of an error in the experiment), denoted as $\eta^{*}$, is plotted in green. Gray area depicts the values of $\eta$ that yield less than $2.5 \%$ below the optimal performance. $\boldsymbol{B}$, Median of the deviations from optimal weighting of previous trials. Dyslexics' deviation was significantly larger than controls' deviation (Mann-Whitney test, $z=2.5, p<0.01$ ). Error bars denote IQR.

previous trials. This suggests a process in which the comparison is preceded by the formation of a combined representation. We hypothesized that if dyslexics' reduced weighting of previous trial stems from an impaired formation of an integrated representation, reduced sensitivity to stimulus statistics may be apparent even before the second tone is presented. To test this hypothesis, we measured ERPs. We focused on the dynamics and magnitude of the $\mathrm{P} 2$ component, which is an automatic response evoked by the auditory cortex (Sheehan et al., 2005; Mayhew et al., 2010). Previous studies, using both oddball (mismatched negativity, MMN; Haenschel et al., 2005; Baldeweg, 2007; Tong et al., 2009) and discrimination paradigms (Tremblay et al., 2001, 2010; Ross and Tremblay, 2009) have shown that the magnitude of this component increases with stimulus repetitions, suggesting that this component is sensitive to the statistics of the experiment. We hypothesized that P2's sensitivity to stimulus repetitions is a special case of its analog sensitivity to the congruency between the current stimulus and the prior distribution. Therefore, we predicted that the magnitude of the control's P2 would be larger in Bias + trials than in Bias - trials, since the average distance of the first tone from the mean frequency was smaller in the Bias + trials than in the Bias - trials (as shown in Fig. 1A,B). Consequently, the first tone in the Bias + trials was more congruent with the prior than the first tone in the Bias - trials. We further predicted that dyslexics' $\mathrm{P} 2$ would not be as sensitive to trial type.

We recorded ERPs under both passive and active conditions in separate sessions. In the passive condition, the same series of stimuli was presented to the participants while they were watching a silent movie and were not asked to perform any task. For each participant in each of the specified trial types, we calculated the area under the curve between 150 and $250 \mathrm{~ms}$ after the first tone's onset as his/her individual P2 area. As predicted, we found that the controls' evoked response (Fig. 5A) was sensitive to the trial type. This was visible in the $\mathrm{P} 2$ component of the response to the first tone. In the Bias + trials, this response was, on average, significantly larger than the response to the first tone of the Biastrials (Wilcoxon test, $p<0.005$ ). However, the dyslexics' P2 was not sensitive to trial type (Fig. $5 B$; Wilcoxon tests, $p=0.46$ ). The difference between controls' and dyslexics' P2 sensitivity to trial type was also significant (Group $\times$ Condition interaction; Man$\mathrm{n}$-Whitney test, $z=2.5, p<0.05)$. We repeated this analysis when participants were asked to actively perform the task with the same stimuli, and found similar results. controls' $\mathrm{P} 2$ was sensitive to the trial type (Fig. $5 C$; Wilcoxon test, $p<0.05$ ), although the magnitude of this effect was smaller than in the passive condition (perhaps due to masking by additional active components). Dyslexics' P2 did not significantly differ between the two trial types (Fig. 5D; Wilcoxon tests, $p=0.67$ ). In this condition, the interaction was not significant (Condition $\times$ Group; MannWhitney test, $z=1.2, p=0.22$ ). Together, these results support the hypothesis that dyslexics' computational deficit is associated with a failure to reliably integrate their on-line representation with the prior distribution.

In principle, we expected that individuals' $\mathrm{P} 2$ sensitivity to the stimulus statistics would be correlated with their weighting of previous trials $(\eta)$. This correlation is expected both if $\mathrm{P} 2$ reflects the result of the process of incorporation of previous trials into current memory (IMM), thus directly reflecting the magnitude of the contribution of previous trials, and if it measures the reliability of the memory trace, which is not captured in the simple IMM model. However, as shown in Figure 5, top right insets, single subject distribution, while at the group level the difference in P2 sensitivity was very consistent, the magnitude of $\mathrm{P} 2$ sensitivity to stimulus statistics was too small (in terms of signal-to-noise ratio) to enable a reliable calculation of these correlations.

\section{Dyslexics' insensitivity to stimulus statistics in a protocol with a reference}

Dyslexics' reduced sensitivity to stimulus statistics leads to nonintuitive predictions on specific trials that should be more challenging for them in other protocols with more structured statistics. We examined the interleaved reference protocol ( $\mathrm{Na}-$ hum et al., 2010). In this protocol, a reference $(1000 \mathrm{~Hz})$ tone is presented on every trial. However, on odd trials it is presented first (Ref first), whereas on even trials it is presented second (Ref second). Intuitively (and consistent with the anchoring deficit hypothesis), dyslexics' performance is expected to be impaired in both, since both types of trials contain a repeated reference. However, the rationale behind the IMM model prompts a specific prediction with respect to different trial types, since previous trials are specifically integrated into the representation of the first tone on each trial. It therefore predicts that controls should benefit substantially from previous statistics in Ref first trials. In these trials, integrating previous statistics reduces the noisiness of the 


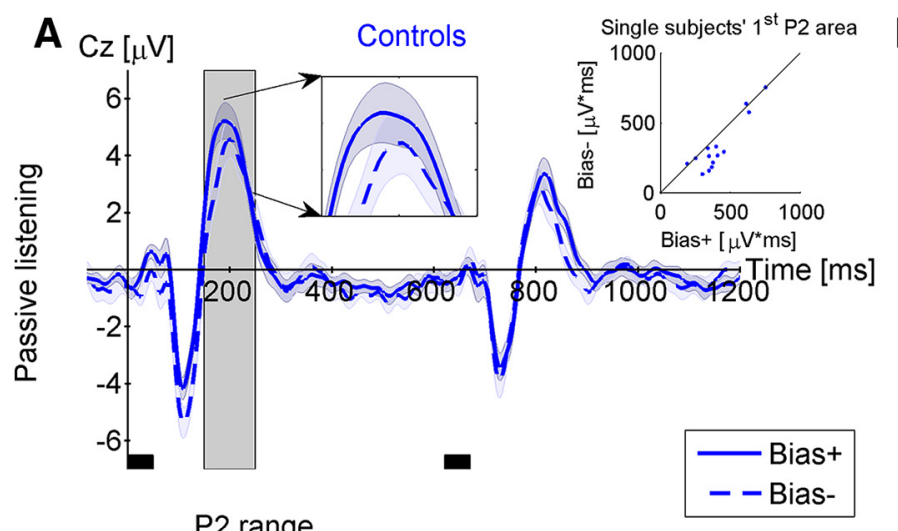

P2 range

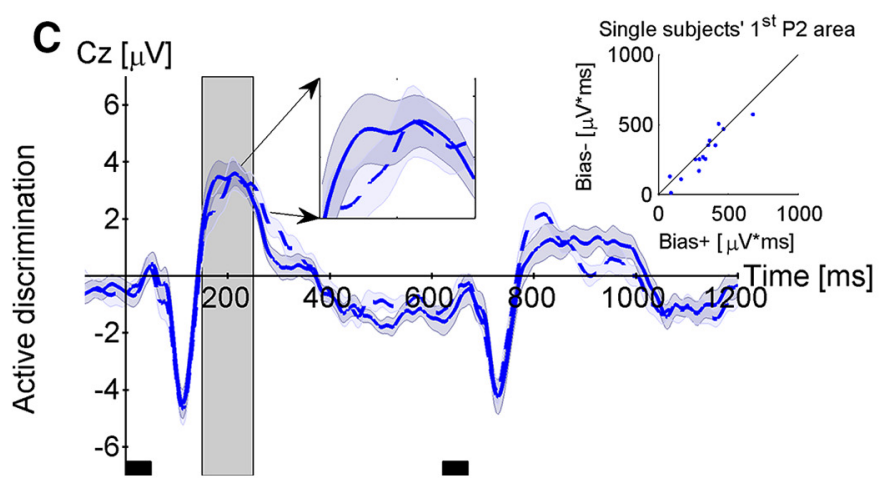

B

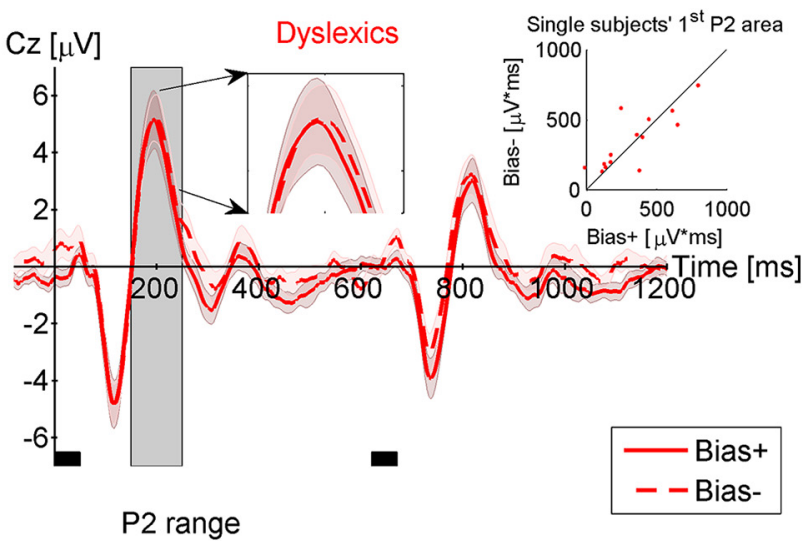

D

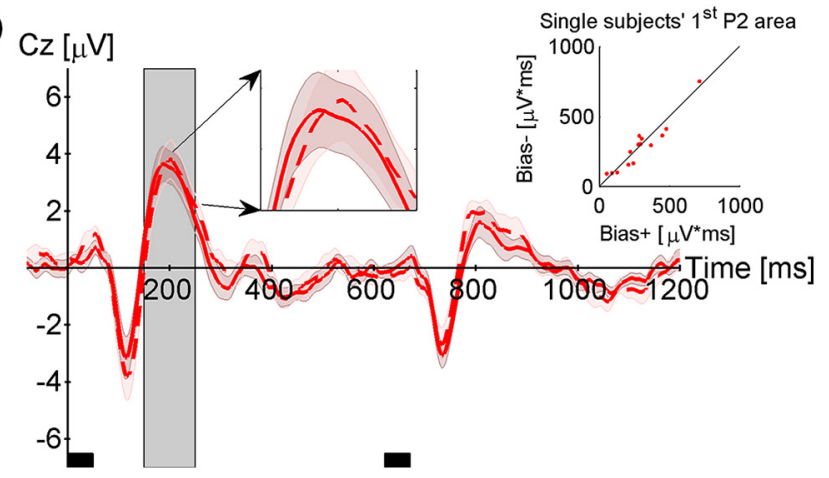

Figure 5. Grand average ERP measures for the Bias + and Bias - trial types (electrode CZ).A, C, Controls (blue lines). B, D, Dyslexics (red lines). Bias + trials are denoted by solid lines and Bias - trials by dashed lines. In Controls, the area of $P 2$ after the first tone (from 150 to $250 \mathrm{~ms}$, denoted by the gray rectangles) was significantly different between Bias + and Bias - trial types, in both Passive Listening $(A)$ and during Active Discrimination (C; Wilcoxon tests, Passive: $p<0.005$; Active: $p<0.05$ ). Dyslexics' evoked responses did not differ between the two trial types (Wilcoxon tests, $\boldsymbol{B}$ Passive: $p=0.46 ; \boldsymbol{D}$ Active: $p=0.67$ ). Controls' P2 to Bias0 trials were in between the Bias + and Bias - responses and are not shown for clarity. Filled areas around the mean response denote cross-subjectSEM. Small black rectangles under the plots denote the temporal location of the two tones in the trial. Insets, Middle of each plot, P2 region enlarged; top right of each plot, single subject data of Bias - versus Bias + trials. In the Passive condition the difference between the trial types was significantly larger among Controls than among Dyslexics (Condition $\times$ Group interaction; Mann-Whitney test for interaction of Bias + vs Bias - and Controls vs Dyslexics, $z=2.5, p<0.05$ ).

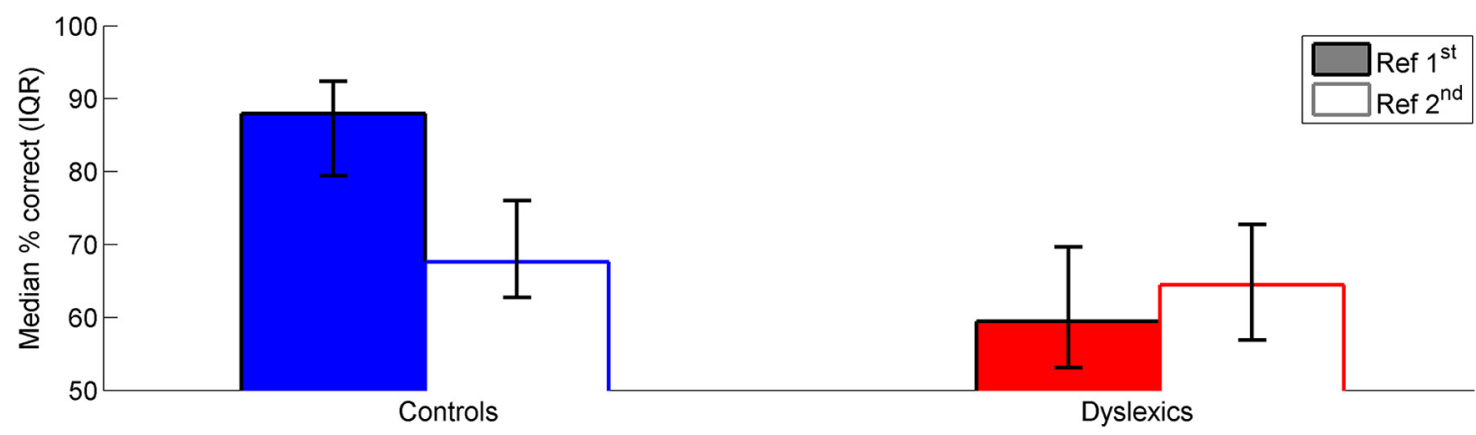

Figure 6. Performance on the two-tone discrimination task using a protocol with a reference frequency $(1000 \mathrm{~Hz})$ presenting the first and second tone in an interleaved manner across trials. Performance in trials in which the reference was presented first (Ref first, filled bars) and in trials in which the reference was presented second (Ref second, empty bars). Bars denote the median of participants' percentage correct. Error bars denote IQR. Controls' performance was more affected by the reference than Dyslexics' performance (Condition $\times$ Group interaction; Mann-Whitney test for interaction between Reference position and Control vs Dyslexics, $z=3, p<0.005)$.

representation of the first tone without modifying its mean. In contrast, in Ref second trials, the second tone is (approximately) equal to the mean of the distribution. Therefore, the incorporation of the prior (approximately the mean) to the representation of the first tone in the same manner decreases the perceived difference and hampers performance. If dyslexics' sensitivity to the statistics of the experiment is indeed reduced, the difference between their performances on the two trial types should be significantly smaller than that of controls.

To test this prediction, all participants performed this interleaved protocol. In line with the IMM prediction, and consistent with previous observations (see also Romo and Salinas,
2003; Nahum et al., 2010), we found that the performance of controls in Ref first trials was substantially and significantly better than their performance in the Ref second trials (Fig. 6, blue; Ref first: mean $86 \pm 2 \%$ SEM correct; Ref second: $69 \pm$ $4 \%$ correct; Wilcoxon test, $p<0.001)$. In contrast, in line with the IMM's prediction, dyslexics' performance did not differ between these trial types (Fig. 6 red; Ref first: $64 \pm 4 \%$ correct; Ref second: $66 \pm 4 \%$ correct; Wilcoxon test, $p=0.71)$. The group difference in sensitivity to trial types was also significant (Condition $\times$ Group interaction; Mann-Whitney test for interaction between Reference position and group, $z=3, p<$ $0.005)$. 


\section{Discussion}

\section{Summary of results}

We dissociated the factors underlying discrimination performance into factors that stem from internal noise within a trial and those that stem from context; i.e., the stimulus statistics. We found that the context effects were significantly and substantially larger in the population of adequate readers, compared with dyslexics. When calculating the optimal weighting of previous trials, given the within-trial noise and the stimulus statistics, we found that dyslexics' weighting but not controls' weighting was indeed significantly lower than optimal. Finally, to tap dyslexics' specific difficulty in adequately weighting previous trials, we recorded ERPs. These recordings suggested that dyslexics' perceptual difficulties are associated with inadequate automatic updating and integrating of the context of the experiment with their current stimulus response.

Dyslexics' specific pattern of reduced sensitivity to the first tone in the pair, as suggested by the IMM model and observed in the ERP measures, also yielded nonintuitive predictions for a novel protocol that motivated the last experiment. Here the reference tone was presented either as the first or as the second tone. The findings showed that dyslexics' difficulties were specific to Ref first trials.

We interpret all these results as an indication that dyslexics (but not controls) underweigh history in perceptual decisions. However, dyslexics' weighting of history could alternatively be close to optimal while their deficit could be associated with increased memory noise (a possibility that goes beyond the IMM, since it requires additional assumptions). In that case, increasing the weight of previous trials may not enhance their performance since it will also increase the memory noise.

\section{Relation to previous studies of dyslexia}

The IMM is an extended formalization of the anchoring hypothesis of dyslexia. This theory was motivated by the observation that controls' better performance in psychoacoustic tasks (McAnally and Stein, 1996; Witton et al., 1998; Ramus et al., 2003; Ahissar et al., 2006; Ahissar, 2007) and in speech discrimination (McArthur and Bishop, 2005; Ahissar et al., 2006; Boets et al., 2007) is restricted, to a large extent, to experiments in which a single reference stimulus or a small range of reference stimuli are used (Ahissar et al., 2006; Ahissar, 2007; Chandrasekaran et al., 2009; Banai and Ahissar, 2010; Oganian and Ahissar, 2012). The theory posits that repeated stimuli serve as an anchor, and thus boost performance when these stimuli are subsequently used. According to this theory, the ability to track such simple regularities is deficient in dyslexia.

The IMM presented here proposes a specific, well-defined computation that is impaired in dyslexics' incorporation of stimulus statistics. As such, the model specifies the conditions where incorporating previous trials is expected to improve perception and those where it is expected to hamper perception. Thus, unlike the intuitive anchoring hypothesis, stimulus repetition is not necessary (e.g., Bias + trials in a protocol with no reference), and may even not be beneficial (e.g., Ref second trials in the reference interleaved protocol). The IMM predicts that dyslexics will only perform worse than controls on the trials that benefit from stimulus statistics.

The IMM assumes that the stimulus statistics are continuously learned and used. We found that this was indeed the case for controls and only to a lesser extent for dyslexics. The ERP recordings further illustrated the analog nature of the automatic tracking mechanisms. The traditional ERP measure of automatic tracking of sounds, the MMN component, is sensitive to the violation of repetition, and is studied in the context of oddball paradigms (Näätänen et al., 1978). The MMN component is often smaller among dyslexics (Baldeweg et al., 1999; Kujala et al., 2003; Bishop, 2007). The findings here show that controls' automatic ERP response is parametrically sensitive to the distance of the stimulus from the peak of the stimulus distribution in previous trials. No such sensitivity was found in the dyslexic population.

The anchoring hypothesis and its computational implementation are inconsistent with the hypothesis that poor phonological representations are the core deficit in dyslexia (Snowling, 2000) for at least two reasons. Not only did we find difficulties in the processing of nonphonological stimuli, but in the computational framework, dyslexics' main difficulty lay in poor usage of context. Specifically they used an overly low $\eta$ given their somewhat higher $\sigma$.

However, our computational model is tightly related to hypotheses that associate dyslexics' difficulties with a failure to make effective predictions that can facilitate task performance ("predictive coding"; Díaz et al., 2012). However, it is also compatible with hypotheses that dyslexics are less resilient to external noise (Sperling et al., 2005, 2006; Beattie et al., 2011; Conlon et al., 2012; Partanen et al., 2012; the "noise exclusion hypothesis"). According to the Bayesian framework underpinning the IMM, the prior information is used to compensate for the noise in the representation of the stimuli. We found that dyslexics do not properly adjust the weight of previous trials to the level of internal noise (Fig. 4). Functionally, this results in reduced noise exclusion. However, putting this broad concept in a computational framework leads to a counterintuitive prediction: when the context is compromising (e.g., Bias - like conditions leading to disruptive predictions), dyslexics should not do worse and may even do better than controls since this mechanism for "noise exclusion" is biased by the prior statistics.

Other studies have suggested that dyslexics' implicit sequence learning is impaired (Vicari et al., 2003; Stoodley et al., 2006; Jiménez-Fernández et al., 2011). This phrasing is very broad. For example, it is not clear whether the representation of syllables should be intact, while the representation of words, which are perhaps formed by implicit sequential learning (conditional probabilities between adjacent syllables; Saffran et al., 1996), should be impaired. Our computational model assumes poor incorporation of basic attributes (zero-order statistics), as observed by the poor usage of the mean frequency of the experiment. It predicts that the same deficit may lead to reduced sensitivity to the prevalence of single syllables.

Other studies (Ramus and Szenkovits, 2008; Boets et al., 2013; Ramus, 2014) have proposed that phonological representations remain intact, but that dyslexics' access to these representations is inefficient, perhaps due to impaired connectivity between the superior temporal areas that encode auditory stimuli and the frontal areas (e.g., Broca) that use them. This hypothesis assumes a clear distinction between representations and access, although it does not specify what "access" is and which retrieval conditions should pose difficulties (e.g., whether implicit priming should be impaired). Our own perspective, as described above, is that perception is never devoid of context. Ease of retrieval is heavily affected by the availability of adequate predictions, which can substantially facilitate the process. Thus, difficulty in using priors may impair the efficiency of retrieval. Nevertheless, the nature of retrieval processes in different behavioral contexts is far from being understood. 
Recently, it was shown that a hierarchical model of perception is able to reconstruct or decode the dynamics underlying generated series of stimuli (Yildiz et al., 2013). Our observations may perhaps be interpreted within this framework, and suggest that dyslexics' deficit resides in the higher and slower level of the hierarchy, which is characterized by larger time constants, or in their impaired communication with lower levels (Boets et al., 2013). The slower dynamics in this higher level are able to track cumulating evidence and form a prior based on the underlying statistics of the stimuli. In turn, these predictions are used to modulate the lower levels of the hierarchy to better match the upcoming stimuli and the requirements of the task. This coincides with data showing that among dyslexics, task-related topdown modulation is impaired (Díaz et al., 2012).

\section{Implications, limits, and future directions}

We posited that inadequate usage of priors in the context of spoken or written language underlies dyslexics' reading deficit. This generalization is based on the fact that proficient reading of single words and even pseudowords, which are impaired in dyslexia, heavily relies on priors related to sound sequences (e.g., phonological, morphological and orthographic; Norris, 2013). In fact, dyslexics' reading of familiar words is more serial (van der Leij and van Daal, 1999; Ziegler et al., 2003; Zoccolotti et al., 2005; Martens and de Jong, 2006), and their "visual word form area," an area of reading expertise that develops in parallel to readers' gradual reliance on word priors, i.e., word familiarity, is not adequately developed (Paulesu et al., 2001; Shaywitz et al., 2002; McCandliss et al., 2003).

However, even within the framework of the simple IMM there are open questions. First, we also found a group difference for within-trial noise $(\sigma)$, which we did not expect, and should be further investigated. Additionally, it is unclear which dimensions of the priors are impaired along with frequency, e.g., intervals (Banai and Ahissar, 2006), or frequency changes in time (Goswami et al., 2011), and at which timescales (the scale of phonemes, syllables, words, or phrases). Moreover, dyslexics' deficit could also include other modalities, such as the spatial distribution of visual stimuli (Moores et al., 2011; Franceschini et al., 2012). A better understanding of these features is particularly important when designing novel training procedures to improve dyslexics' reading skills.

\section{References}

Ahissar M (2007) Dyslexia and the anchoring-deficit hypothesis. Trends Cogn Sci 11:458-465. CrossRef Medline

Ahissar M, Lubin Y, Putter-Katz H, Banai K (2006) Dyslexia and the failure to form a perceptual anchor. Nat Neurosci 9:1558-1564. CrossRef Medline

Alcalá-Quintana R, García-Pérez MA (2011) A model for the time-order error in contrast discrimination. Q J Exp Psychol 64:1221-1248. CrossRef Medline

Amitay S, Ben-Yehudah G, Banai K, Ahissar M (2002) Disabled readers suffer from visual and auditory impairments but not from a specific magnocellular deficit. Brain 125:2272-2285. CrossRef Medline

Ashourian P, Loewenstein Y (2011) Bayesian inference underlies the contraction bias in delayed comparison tasks. PLoS One 6:e19551. CrossRef Medline

Baldeweg T (2007) ERP repetition effects and mismatch negativity generation. J Psychophysiol 21:204-213. CrossRef

Baldeweg T, Richardson A, Watkins S, Foale C, Gruzelier J (1999) Impaired auditory frequency discrimination in dyslexia detected with mismatch evoked potentials. Ann Neurol 45:495-503. CrossRef Medline

Banai K, Ahissar M (2006) Auditory processing deficits in dyslexia: task or stimulus related? Cereb Cortex 16:1718-1728. CrossRef Medline

Banai K, Ahissar M (2010) On the importance of anchoring and the conse- quences of its impairment in dyslexia. Dyslexia 16:240-257. CrossRef Medline

Banai K, Ahissar M (2013) Musical experience, auditory perception and reading-related skills in children. PLoS One 8:e75876. CrossRef Medline

Banai K, Yifat R (2012) Auditory working memory and early reading skills in Hebrew-speaking preschool children. J Basic Clin Physiol Pharmacol 23:109-115. CrossRef Medline

Beattie RL, Lu ZL, Manis FR (2011) Dyslexic adults can learn from repeated stimulus presentation but have difficulties in excluding external noise. PLoS One 6:e27893. CrossRef Medline

Ben-Yehudah G, Banai K, Ahissar M (2004) Patterns of deficit in auditory temporal processing among dyslexic adults. Neuroreport 15:627-631. Medline

Bishop DV (2007) Using mismatch negativity to study central auditory processing in developmental language and literacy impairments: where are we, and where should we be going? Psychol Bull 133:651-672. CrossRef Medline

Boets B, Wouters J, van Wieringen A, Ghesquière P (2007) Auditory processing, speech perception and phonological ability in pre-school children at high-risk for dyslexia: a longitudinal study of the auditory temporal processing theory. Neuropsychologia 45:1608-1620. CrossRef Medline

Boets B, Op de Beeck HP, Vandermosten M, Scott SK, Gillebert CR, Mantini D, Bulthé J, Sunaert S, Wouters J, Ghesquière P (2013) Intact but less accessible phonetic representations in adults with dyslexia. Science 342: 1251-1254. CrossRef Medline

Chandrasekaran B, Hornickel J, Skoe E, Nicol T, Kraus N (2009) Contextdependent encoding in the human auditory brainstem relates to hearing speech in noise: implications for developmental dyslexia. Neuron 64:311319. CrossRef Medline

Conlon EG, Lilleskaret G, Wright CM, Power GF (2012) The influence of contrast on coherent motion processing in dyslexia. Neuropsychologia 50:1672-1681. CrossRef Medline

Deutsch A, Bentin S (1996) Attention factors mediating syntactic deficiency in reading-disabled children. J Exp Child Psychol 63:386-415. CrossRef Medline

Díaz B, Hintz F, Kiebel SJ, von Kriegstein K (2012) Dysfunction of the auditory thalamus in developmental dyslexia. Proc Natl Acad Sci U S A 109:13841-13846. CrossRef Medline

Franceschini S, Gori S, Ruffino M, Pedrolli K, Facoetti A (2012) A causal link between visual spatial attention and reading acquisition. Curr Biol 22:814-819. CrossRef Medline

Goswami U, Fosker T, Huss M, Mead N, Szucs D (2011) Rise time and formant transition duration in the discrimination of speech sounds: the Ba-Wa distinction in developmental dyslexia. Dev Sci 14:34-43. CrossRef Medline

Haenschel C, Vernon DJ, Dwivedi P, Gruzelier JH, Baldeweg T (2005) Event-related brain potential correlates of human auditory sensory memory-trace formation. J Neurosci 25:10494-10501. CrossRef Medline

Hairston IS, Nagarajan SS (2007) Neural mechanisms of the time-order error: an MEG study. J Cogn Neurosci 19:1163-1174. CrossRef Medline

Hornickel J, Kraus N (2013) Unstable representation of sound: a biological marker of dyslexia. J Neurosci 33:3500-3504. CrossRef Medline

Jiménez-Fernández G, Vaquero JM, Jiménez L, Defior S (2011) Dyslexic children show deficits in implicit sequence learning, but not in explicit sequence learning or contextual cueing. Ann Dyslexia 61:85-110. CrossRef Medline

Kendall MG, Stuart A, Ord JK (1987) Advanced theory of statistics, ed 5. London: Charles Griffin.

Kujala T, Belitz S, Tervaniemi M, Näätänen R (2003) Auditory sensory memory disorder in dyslexic adults as indexed by the mismatch negativity. Eur J Neurosci 17:1323-1327. CrossRef Medline

Lu ZL, Williamson SJ, Kaufman L (1992) Behavioral lifetime of human auditory sensory memory predicted by physiological measures. Science 258: 1668-1670. CrossRef Medline

MacKay DG (1970) Spoonerisms: the structure of errors in the serial order of speech. Neuropsychologia 8:323-350. CrossRef Medline

Martens VE, de Jong PF (2006) The effect of word length on lexical decision in dyslexic and normal reading children. Brain Lang 98:140-149. CrossRef Medline

Mayhew SD, Dirckx SG, Niazy RK, Iannetti GD, Wise RG (2010) EEG sig- 
natures of auditory activity correlate with simultaneously recorded fMRI responses in humans. Neuroimage 49:849-864. CrossRef Medline

McAnally KI, Stein JF (1996) Auditory temporal coding in dyslexia. Proc Biol Sci 263:961-965. CrossRef Medline

McArthur GM, Bishop DV (2005) Speech and non-speech processing in people with specific language impairment: a behavioural and electrophysiological study. Brain Lang 94:260-273. CrossRef Medline

McCandliss BD, Cohen L, Dehaene S (2003) The visual word form area: expertise for reading in the fusiform gyrus. Trends Cogn Sci 7:293-299. CrossRef Medline

Mengler ED, Hogben JH, Michie P, Bishop DV (2005) Poor frequency discrimination is related to oral language disorder in children: a psychoacoustic study. Dyslexia 11:155-173. CrossRef Medline

Micheyl C, Delhommeau K, Perrot X, Oxenham AJ (2006) Influence of musical and psychoacoustical training on pitch discrimination. Hear Res 219:36-47. CrossRef Medline

Möller J, Jansma BM, Rodriguez-Fornells A, Münte TF (2007) What the brain does before the tongue slips. Cereb Cortex 17:1173-1178. CrossRef Medline

Moores E, Cassim R, Talcott JB (2011) Adults with dyslexia exhibit large effects of crowding, increased dependence on cues, and detrimental effects of distractors in visual search tasks. Neuropsychologia 49:38813890. CrossRef Medline

Näätänen R, Gaillard AWK, Mäntysalo S (1978) Early selective-attention effects on evoked potential reinterpreted. Acta Psychol 42:313-329. CrossRef Medline

Nagarajan S, Mahncke H, Salz T, Tallal P, Roberts T, Merzenich MM (1999) Cortical auditory signal processing in poor readers. Proc Natl Acad Sci U S A 96:6483-6488. CrossRef Medline

Nahum M, Daikhin L, Lubin Y, Cohen Y, Ahissar M (2010) From comparison to classification: a cortical tool for boosting perception. J Neurosci 30:1128-1136. CrossRef Medline

Norris D (2013) Models of visual word recognition. Trends Cogn Sci 17: 517-524. CrossRef Medline

Oganian Y, Ahissar M (2012) Poor anchoring limits dyslexics' perceptual, memory, and reading skills. Neuropsychologia 50:1895-1905. CrossRef Medline

Parbery-Clark A, Strait DL, Kraus N (2011) Context-dependent encoding in the auditory brainstem subserves enhanced speech-in-noise perception in musicians. Neuropsychologia 49:3338-3345. CrossRef Medline

Partanen M, Fitzpatrick K, Mädler B, Edgell D, Bjornson B, Giaschi DE (2012) Cortical basis for dichotic pitch perception in developmental dyslexia. Brain Lang 123:104-112. CrossRef Medline

Paulesu E, Démonet JF, Fazio F, McCrory E, Chanoine V, Brunswick N, Cappa SF, Cossu G, Habib M, Frith CD, Frith U (2001) Dyslexia: cultural diversity and biological unity. Science 291:2165-2167. CrossRef Medline

Preuschhof C, Schubert T, Villringer A, Heekeren HR (2010) Prior Information biases stimulus representations during vibrotactile decision making. J Cogn Neurosci 22:875-887. CrossRef Medline

Ramus F (2014) Neuroimaging sheds new light on the phonological deficit in dyslexia. Trends Cogn Sci 18:274-275. CrossRef Medline

Ramus F, Ahissar M (2012) Developmental dyslexia: the difficulties of interpreting poor performance, and the importance of normal performance. Cogn Neuropsychol 29:104-122. CrossRef Medline

Ramus F, Szenkovits G (2008) What phonological deficit? Q J Exp Psychol 61:129-141. CrossRef Medline

Ramus F, Rosen S, Dakin SC, Day BL, Castellote JM, White S, Frith U (2003) Theories of developmental dyslexia: insights from a multiple case study of dyslexic adults. Brain 126:841-865. CrossRef Medline

Raviv O, Ahissar M, Loewenstein Y (2012) How recent history affects perception: the normative approach and its heuristic approximation. PLoS Comput Biol 8:e1002731. CrossRef Medline

Romo R, Salinas E (2003) Flutter discrimination: neural codes, perception, memory and decision making. Nat Rev Neurosci 4:203-218. CrossRef Medline
Romo R, Hernández A, Zainos A, Lemus L, Brody CD (2002) Neuronal correlates of decision-making in secondary somatosensory cortex. Nat Neurosci 5:1217-1225. CrossRef Medline

Ross B, Tremblay K (2009) Stimulus experience modifies auditory neuromagnetic responses in young and older listeners. Hear Res 248:48-59. CrossRef Medline

Saffran JR, Aslin RN, Newport EL (1996) Statistical learning by 8-monthold infants. Science 274:1926-1928. CrossRef Medline

Shaywitz BA, Shaywitz SE, Pugh KR, Mencl WE, Fulbright RK, Skudlarski P, Constable RT, Marchione KE, Fletcher JM, Lyon GR, Gore JC (2002) Disruption of posterior brain systems for reading in children with developmental dyslexia. Biol Psychiatry 52:101-110. CrossRef Medline

Sheehan KA, McArthur GM, Bishop DV (2005) Is discrimination training necessary to cause changes in the $\mathrm{P} 2$ auditory event-related brain potential to speech sounds? Brain Res Cogn Brain Res 25:547-553. CrossRef Medline

Sheppard WF (1899) On the application of the theory of error to cases of normal distribution and normal correlation. Philos Trans R Soc Lond 192:101-167. CrossRef

Shteingart H, Neiman T, Loewenstein Y (2013) The role of first impression in operant learning. J Exp Psychol Gen 142:476-488. CrossRef Medline Snowling MJ (2000) Dyslexia. Wiley-Blackwell.

Sperling AJ, Lu ZL, Manis FR, Seidenberg MS (2005) Deficits in perceptual noise exclusion in developmental dyslexia. Nat Neurosci 8:862-863. CrossRef Medline

Sperling AJ, Lu ZL, Manis FR, Seidenberg MS (2006) Motion-perception deficits and reading impairment: it's the noise, not the motion. Psychol Sci 17:1047-1053. CrossRef Medline

Stoodley CJ, Harrison EP, Stein JF (2006) Implicit motor learning deficits in dyslexic adults. Neuropsychologia 44:795-798. CrossRef Medline

Tong Y, Melara RD, Rao A (2009) P2 enhancement from auditory discrimination training is associated with improved reaction times. Brain Res 1297:80-88. CrossRef Medline

Tremblay K, Kraus N, McGee T, Ponton C, Otis B (2001) Central auditory plasticity: changes in the N1-P2 complex after speech-sound training. Ear Hear 22:79-90. CrossRef Medline

Tremblay KL, Inoue K, McClannahan K, Ross B (2010) Repeated stimulus exposure alters the way sound is encoded in the human brain. PLoS One 5:e10283. CrossRef Medline

van der Leij A, van Daal VH (1999) Automatization aspects of dyslexia speed limitations in word identification, sensitivity to increasing task demands, and orthographic compensation. J Learn Disabil 32:417-428. CrossRef Medline

Vicari S, Marotta L, Menghini D, Molinari M, Petrosini L (2003) Implicit learning deficit in children with developmental dyslexia. Neuropsychologia 41:108-114. CrossRef Medline

Walter WG, Cooper R, Aldridge VJ, Mccallum WC, Winter a L (1964) Contingent negative variation: an electric sign of sensorimotor association and expectancy in the human brain. Nature 203:380-384. CrossRef Medline

Wechsler D (1997) Wechsler Adult Intelligence Scale (WAIS-III) Administration and Scoring Manual. San Antonio, TX: The Psychological Corporation.

Witton C, Talcott JB, Hansen PC, Richardson AJ, Griffiths TD, Rees A, Stein JF, Green GG (1998) Sensitivity to dynamic auditory and visual stimuli predicts nonword reading ability in both dyslexic and normal readers. Curr Biol 8:791-797. CrossRef Medline

Yildiz IB, von Kriegstein K, Kiebel SJ (2013) From birdsong to human speech recognition: Bayesian inference on a hierarchy of nonlinear dynamical systems. PLoS Comput Biol 9: e1003219. CrossRef Medline

Ziegler JC, Perry C, Ma-Wyatt A, Ladner D, Schulte-Körne G (2003) Developmental dyslexia in different languages: language-specific or universal? J Exp Child Psychol 86:169-193. CrossRef Medline

Zoccolotti P, De Luca M, Di Pace E, Gasperini F, Judica A, Spinelli D (2005) Word length effect in early reading and in developmental dyslexia. Brain Lang 93:369-373. CrossRef Medline 